\title{
Research Article \\ Sustained Release of Green Tea Polyphenols from Liposomal Nanoparticles; Release Kinetics and Mathematical Modelling
}

\author{
Ravi Theaj Prakash Upputuri, Abul Kalam Azad Mandal* \\ School of Bio Sciences and Technology, VIT University, Vellore-632014, Tamil Nadu, India \\ ${ }^{*}$ Corresponding author: Abul Kalam Azad Mandal, School of Bio Sciences and Technology, VIT University, Vellore-632014, Tamil Nadu, India. \\ Tel: +91 9443758596, E-mail: akazadmandal@vit.ac.in
}

Received: 21 Agust 2015; Revised: 30 August 2017; $\quad$ Accepted: 8 October 2017; $\quad$ Published online: 29 December 2017

\begin{abstract}
Background: Green tea polyphenols (GTP) are known to have several health benefits. In spite of these benefits, its application as a therapeutic agent is limited due to some of its limitations such as stability, bioavailability, and biotransformation. To overcome these limitations, liposomal nanoparticles have been used as a carrier of the GTP.

Objective: Encapsulation of GTP to the liposomal nanoparticles in order to achieve a sustained release of the GTP and to determine the drug release kinetics and the mechanism of the release.

Materials and Methods: GTP encapsulated liposomal nanoparticles were prepared using phosphatidyl choline and cholesterol. The synthesized particles were characterized for their particle size and morphology. In vitro release studies were carried out, followed by drug release kinetics, and determining the mechanism of release. In vitro, antioxidant assay was determined following 2,2-diphenyl-1-picrylhydrazyl (DPPH) method.

Results: Atomic force microscope (AFM) and high resolution scanning electron microscope (HR SEM) images showed spherical particles of the size of 64.5 and $252 \mathrm{~nm}$. An encapsulation efficiency as high as $77.7 \%$ was observed with GTP concentration of $5 \mathrm{mg} \cdot \mathrm{mL}^{-1}$. In vitro release studies showed that the loading concentrations of GTP were independent to the cumulative percentage of the drug release. GTP release by varying the $\mathrm{pH}$ and temperature showed a direct correlation between the release parameter and the percentage of drug release. The higher the $\mathrm{pH}$ and temperature, the higher was the percentage of the drug release. The release data showed a good correlation with Zero order kinetics and the mechanism of the release being anomalous mode. Radical scavenging activity of the released GTP showed a potent scavenging activity.

Conclusion: GTP encapsulated liposomal nanoparticles could be used as a delivery vehicle for achieving a sustained release.

Keywords: Anomalous transport, Diffusion, Erosion, Sustained release, Zero order kinetics.
\end{abstract}

\section{Background}

Liposomes are considered to be an ideal drug carrier as they consist of an aqueous core encapsulated by the natural or synthetic phospholipids and can deliver both hydrophobic and hydrophilic drugs. Hydrophobic drugs are likely to be entrapped within the lipid bilayer, while the hydrophilic drugs have a tendency to be entrapped within the core (1). Several liposomal formulations have been approved for delivery of the drugs like amphotericin B (2), doxorubicin (3), vincristine sulfate (4), cytarabine (5), etc. Few of the recent advancements in the liposomal nanoparticles based drug delivery system include the work of Ong et al. (6), where liposomal ciprofloxacin have been explored to predict the enhanced lung residence time of the formulation.
Zhou et al. (2015) developed a novel liposomal formulation for the cisplatin to study the potent antitumor activity in vitro and in vivo and achieved significantly decreased the incidence of the side effects with cisplatin loaded liposomal nanoparticles than that of free cisplatin (7).

The green tea polyphenols (GTP) are known to have a wide range of health benefits. The major constituents of the tea polyphenols, known as catechins constitute about $30-42 \%$ of the dry weight (8). Several reports are available on the applications of GTP in the preventive effects of cancer (9), cardiovascular diseases (10), and neuroprotective effects (11). In spite of several health benefits, its use as a therapeutic agent is limited due to a few limitations such as bioavailability, stability, 
and biotransformation $(12,13)$. To overcome these limitations and improve the efficiency of GTP, liposomal nanoparticles have been developed and used as a carrier for the delivery of GTP.

\section{Objectives}

The present study deals with the encapsulation of GTP to the liposomal nanoparticles to achieve sustained release of the GTP, to determine the drug release kinetics, and the mechanism of the release.

\section{Materials and Methods}

The green tea polyphenols (GTP) was purchased from the Sigma Chemical Co., USA. Other chemicals such as cholesterol, phosphatidylcholine, and tween 80 were procured from Himedia, India.

\subsection{Synthesis of the GTP Loaded Liposomal Nanoparticles}

Phosphatidyl choline and cholesterol in a ratio of 20:1 was dissolved in $2 \mathrm{~mL}$ ethanol to form a lipid phase. This mixture was heated in a water bath at $60{ }^{\circ} \mathrm{C}$. Tween $80(0.1 \%)$ was dissolved in $10 \mathrm{~mL}$ PBS (pH $6.5)$ to form an aqueous phase. The obtained aqueous phase was dropped into lipid phase upon stirring. The mixture was placed on a magnetic stirrer for $10 \mathrm{~min}$ followed by sonication at $30 \%$ amplification for 10 $\mathrm{min}$. The final liposomal nanoparticles were obtained after being cooled in the ice bath, diluted to $20 \mathrm{~mL}$, and filtered. For GTP loaded liposomal nanoparticles, different quantities $(1,2.5,5$ and $10 \mathrm{mg})$ of the GTP were dissolved in $1 \mathrm{~mL}$ ethanol prior to the dissolution of the phosphatidyl choline and cholesterol (14).

\subsection{Characterization of Liposomal Nanoparticles}

The hydrodynamic diameter and the surface charge of the nanoparticles were determined by dynamic light scattering (DLS) and $\zeta$ potential analysis using Nanopartica, Nanoparticle analyzer SZ-100. The morphology and polydispersity of the particles were analyzed through atomic force microscope (AFM, Nano Surf Easy Scan2, Switzerland) and high resolution scanning electron microscope (HR SEM, FEI Quanta FEG 200).

\subsection{Determination of Encapsulation Efficiency (EE)}

The encapsulation efficiency was determined by separating the unloaded GTP by centrifugation. The amount of unloaded GTP was quantified by photometric Folin- Ciocalteu assay as described by Swain and Hillis (15). About $0.5 \mathrm{~mL}$ of sample was added to $0.5 \mathrm{~mL}$ of $1 \mathrm{M}$ Follin - Ciocalteu reagent and $0.5 \mathrm{~mL}$ of $35 \%$ sodium carbonate. The mixture was incubated for 30 min in dark and the absorbance was measured at 700 $\mathrm{nm}$ applying a spectrophotometer. EE was calculated using the formula:

$E E(\%)=\{($ Total amount of GTP - Amount of free GTP $)\}$ Total amount of GTP $\} \times 100$

\subsection{Release of GTP from the Liposomal Nanoparticles} Release studies were carried out in the phosphate buffer saline (PBS) at a physiological $\mathrm{pH}$ of 7.4 at $37{ }^{\circ} \mathrm{C}$ through dialysis method. One $\mathrm{mL}$ of the encapsulated GTP nanoparticles was transferred to a dialysis bag with both ends sealed. The bag was placed in a beaker containing $50 \mathrm{~mL}$ of PBS. At appropriate time intervals $(1,2,3,4,5,6,7,8,12,24$, and $48 \mathrm{~h}), 1 \mathrm{~mL}$ of the sample from the beaker was withdrawn. The collected samples were used to determine the amount of drug released. Quantification of the GTP was carried out by the method developed earlier (15). The drug release profiles were studied for the different loading concentrations of GTP $\left(1,2.5,5\right.$, and $\left.10 \mathrm{mg} \cdot \mathrm{mL}^{-1}\right)$ at different $\mathrm{pH}(3,7.4$, and 9) and temperatures (room temperature (RT), at 37 and $45^{\circ} \mathrm{C}$, respectively).

\subsection{In vitro Antioxidant Assay}

The radical scavenging activity of the released GTP was determined in vitro by 1, 1-diphenyl-2-picrylhydrazyl (DPPH) assay following the method of Williams et al. (16). About $0.1 \mathrm{mM}$ DPPH was used for the study and was prepared in the absolute ethanol. To $0.5 \mathrm{~mL}$ of DPPH, $100 \mu \mathrm{L}$ of the sample and $550 \mu \mathrm{L}$ PBS was added. The mixture was incubated for $30 \mathrm{~min}$ in dark and the absorbance was measured at $517 \mathrm{~nm}$ using a spectrophotometer.

\subsection{Mathematical Modelling}

In vitro release data was fitted to various mathematical models to study the drug release kinetics and the mechanism of the drug release was studied as well.. The different models that were used in this study are Zero order, First order, Higuchi, Hixson-Crowell and Korsemeyer-Peppas models (17). The correlation coefficient $\left(\mathrm{R}^{2}\right)$ and the release exponent $(\mathrm{n})$ were used to determine the best-fit kinetic model and the mechanism of the drug release.

\subsection{Statistical Analysis}

All the data were subjected to the analysis of variance (ANOVA) and presented as mean $\pm \mathrm{SD}$. The means were separated by Duncan's multiple range test (DMRT) at $p \leq 0.05$. 


\section{Results}

GTP at different concentrations was encapsulated into the liposomal nanoparticles. The mean hydrodynamic diameter of the particles was observed to be $322.1 \mathrm{~nm}$ with a polydispersity index of 0.921 . The AFM and HR SEM images revealed spherical particles with sizes of 64.5 and $252 \mathrm{~nm}$, respectively (Fig. 1). The particles were negatively charged with a zeta potential value of $-24.2 \pm 2.75 \mathrm{mV}$.
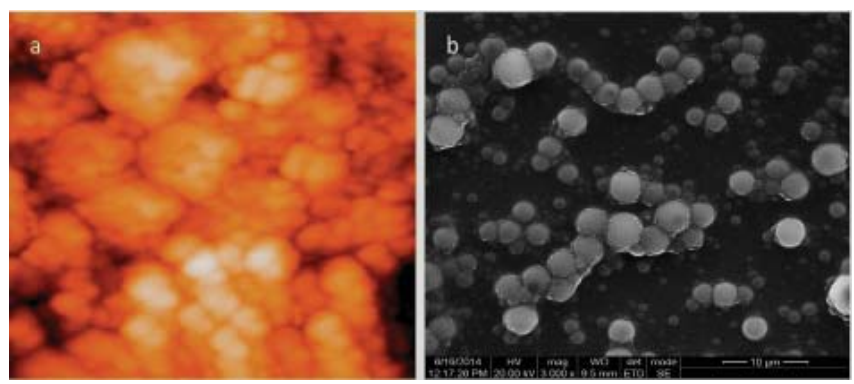

Figure 1. AFM (a) and HR SEM (b) images of the liposomal nanoparticles. The morphology of the particles was observed to be spherical.

The encapsulation efficiency (EE) of the GTP loaded liposomal nanoparticles are presented in Table 1. The maximum EE (77.78\%) was observed when GTP was loaded at a concentration of $5 \mathrm{mg} \cdot \mathrm{mL}^{-1}$.
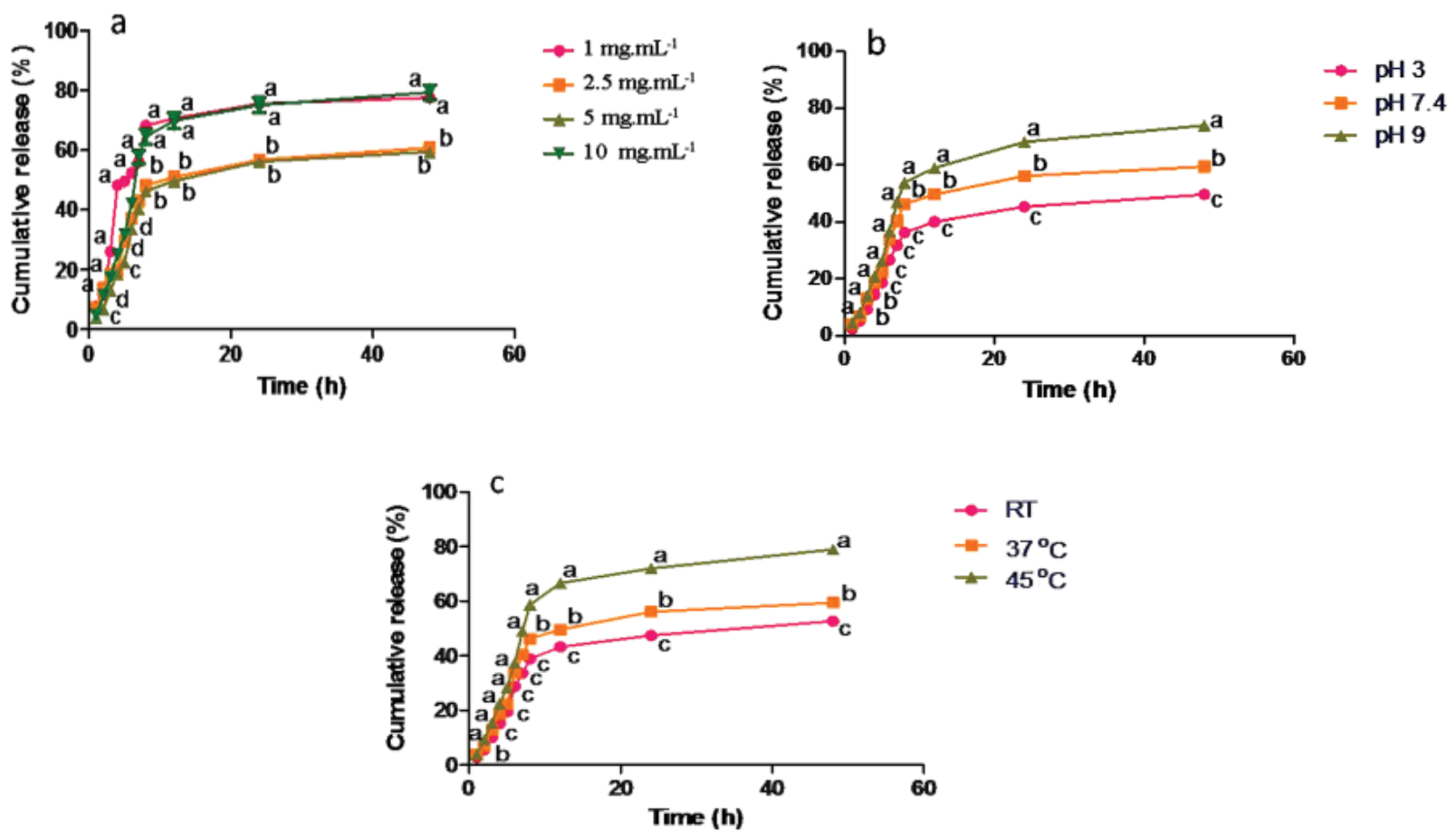

Figure 2. In vitro release of the GTP from liposomal nanoparticles at different concentrations of the GTP (a); different $\mathrm{pH}$ (b) and the different temperatures (c) at the different time intervals. Different letters at a particular time indicate significant difference according to DMRT, $p \leq 0.05(n=3)$. 
the GTP concentration was increased to $10 \mathrm{mg} \cdot \mathrm{mL}^{-1}$, a significant increase in the cumulative percentage of the drug release was observed, where $83 \%$ of the drug was released in $48 \mathrm{~h}$. The release of GTP was monitored by varying $\mathrm{pH}$ of the release medium. It was observed that, as $\mathrm{pH}$ of the medium shifted from acidic to basic, there was an increase in the cumulative percentage of the drug release. About $50 \%$ of the drug was released at pH 3 in 48 h, while at pH 7.4 and 9 it was 60 and 74\%, respectively. As the temperature was increased from RT to $45^{\circ} \mathrm{C}$, an increase in the cumulative percentage of the drug release was observed. At RT, about $53 \%$ of the drug was released within $48 \mathrm{~h}$. As the temperature was increased to $37^{\circ} \mathrm{C}$ and $45^{\circ} \mathrm{C}, 60 \%$ and $79 \%$ of the drug was released within $48 \mathrm{~h}$.

The obtained in vitro release data was fitted to various kinetic models. The regression parameters (i.e., the regression coefficients $\left(\mathrm{R}^{2}\right)$ ), the release kinetic constants (k), and the release exponent (n) are presented in Table 2 for the different parameters. All the release data showed a good correlation with Zero order kinetics. Predominance was observed in the anomalous mode of the drug transport, as the release exponent (n) for all the release data were observed to be in the range of 0.430.89 . The release mechanism here is characterized by coupling of the diffusion and erosion mechanisms.

All the released samples showed potent antioxidant activities. The free radical scavenging activity of GTP loaded liposomal nanoparticles is shown in Figure 3 . The highest scavenging activity was observed to be $75 \%$ for GTP concentration of $10 \mathrm{mg} \cdot \mathrm{mL}^{-1}$ for the sample released in $7 \mathrm{~h}$, while the activity was observed to be $72 \%$ for the GTP concentration of $5 \mathrm{mg} \cdot \mathrm{mL}^{-1} \mathrm{No}$ significant difference in the activity was observed for the GTP concentrations of 2.5 and $1 \mathrm{mg} \cdot \mathrm{mL}^{-1}$.

\section{Discussion}

Several studies have been carried out using liposomes

Table 2. The GTP release profiles with various models and mechanism of release at different conditions.

\begin{tabular}{|c|c|c|c|c|c|c|c|c|c|c|c|}
\hline \multirow{2}{*}{ Condition } & \multicolumn{2}{|c|}{ Zero order } & \multicolumn{2}{|c|}{ First order } & \multicolumn{2}{|c|}{ Higuchi model } & \multicolumn{2}{|c|}{$\begin{array}{c}\text { Hixson-Crowell } \\
\text { model }\end{array}$} & \multicolumn{2}{|c|}{$\begin{array}{l}\text { Korsmeyer- } \\
\text { Peppas model }\end{array}$} & \multirow{2}{*}{$\begin{array}{c}\text { Drug } \\
\text { transport } \\
\text { mechanism }\end{array}$} \\
\hline & $\mathbf{R}^{2}$ & $\mathbf{k}_{0}$ & $\mathbf{R}^{2}$ & $k_{1}$ & $\mathbf{R}^{2}$ & $\mathbf{k}_{\mathrm{H}}$ & $\mathbf{R}^{2}$ & $\mathbf{k}_{\mathrm{HC}}$ & $\mathbf{R}^{2}$ & $\mathbf{N}$ & \\
\hline $\begin{array}{c}1 \mathrm{mg} \cdot \mathrm{mL}^{-1} \\
\mathrm{GTP}\end{array}$ & 0.9337 & 0.615 & 0.9782 & 0.006 & 0.6543 & 9.528 & 0.9724 & 0.014 & 0.7436 & 0.6125 & Anomalous \\
\hline $\begin{array}{c}2.5 \mathrm{mg} \cdot \mathrm{mL}^{-1} \\
\mathrm{GTP}\end{array}$ & 0.9882 & 0.617 & 0.9873 & 0.007 & 0.750 & 10.698 & 0.990 & 0.022 & 0.8461 & 0.5739 & Anomalous \\
\hline $\begin{array}{c}5 \mathrm{mg} \cdot \mathrm{mL}^{-1} \\
\text { GTP }\end{array}$ & 0.9896 & 0.889 & 0.9855 & 0.012 & 0.7543 & 13.783 & 0.9891 & 0.040 & 0.8260 & 0.7617 & Anomalous \\
\hline $\begin{array}{l}10 \mathrm{mg} \cdot \mathrm{mL}^{-1} \\
\text { GTP }\end{array}$ & 0.9783 & 1.401 & 0.9580 & 0.009 & 0.7154 & 24.277 & 0.9720 & 0.036 & 0.8223 & 0.7720 & Anomalous \\
\hline $\mathrm{pH} 3$ & 0.9951 & 0.829 & 0.9876 & 0.012 & 0.7854 & 12.846 & 0.9918 & 0.041 & 0.8252 & 0.8576 & Anomalous \\
\hline $\mathrm{pH} 7.4$ & 0.9896 & 0.889 & 0.9855 & 0.012 & 0.7543 & 13.782 & 0.9891 & 0.040 & 0.8260 & 0.7617 & Anomalous \\
\hline $\mathrm{pH} 9$ & 0.9926 & 0.692 & 0.9612 & 0.009 & 0.7939 & 11.986 & 0.9758 & 0.032 & 0.8511 & 0.7799 & Anomalous \\
\hline RT & 0.9935 & 0.816 & 0.9878 & 0.012 & 0.7741 & 12.653 & 0.9912 & 0.041 & 0.8333 & 0.7903 & Anomalous \\
\hline $37^{\circ} \mathrm{C}$ & 0.9896 & 0.889 & 0.9855 & 0.012 & 0.7543 & 13.782 & 0.9891 & 0.040 & 0.8260 & 0.7617 & Anomalous \\
\hline $45^{\circ} \mathrm{C}$ & 0.9923 & 0.768 & 0.9545 & 0.009 & 0.7815 & 13.302 & 0.9865 & 0.032 & 0.8419 & 0.8099 & Anomalous \\
\hline
\end{tabular}

R: Correlation coefficient; k: Constant; n: Release exponent 


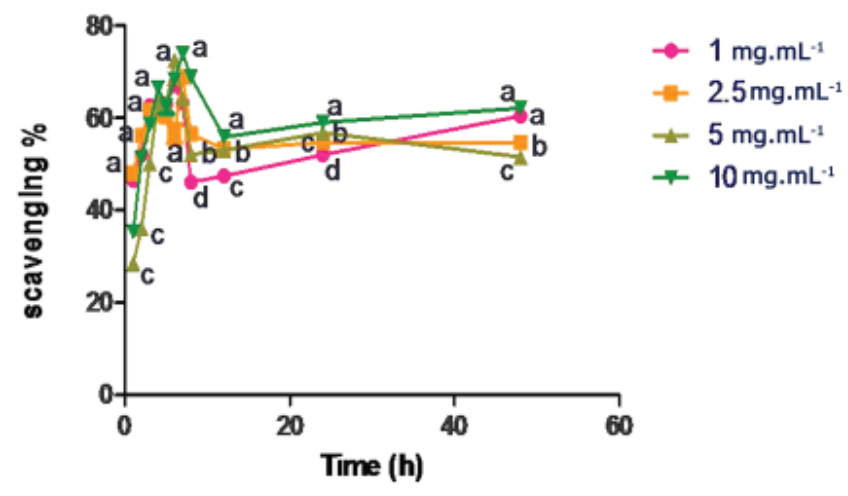

Figure 3. The free radical scavenging activity of the released samples upon loading different concentrations of the GTP to the liposomal nanoparticles. Different letters at a particular time indicate significant difference according to the DMRT, $p \leq 0.05(\mathrm{n}=3)$.

for the drug delivery to the target cancer (18-20), for the treatment of the Alzheimer's disease (21), and various biomedical fields including immunoassay and targeted drug delivery (22). To the best of our knowledge, there are no articles available on the encapsulation of GTP to liposomal nanoparticles. Our work emphasizes on the encapsulation of the GTP to liposomal nanoparticles and developing a sustained release system. Liposomal nanoparticles have been synthesized and the results of our study showed particles with a diameter of $\sim 250$ $\mathrm{nm}$. As well, the particles are being stable ( $\zeta$ potential value of $-24.2 \pm 2.75 \mathrm{mV}$ ), while Wang et al. (23) have reported the surface charge of the liposomal nanoparticles to be $-15 \mathrm{mV}$. The high $\zeta$ potential (positive or negative) provides a good dispersion stability by preventing aggregation and fusion of the liposomes due to electrostatic repulsion (24). The DLS results showed a higher hydrodynamic diameter of the particles compared to that of AFM and HR SEM ( 64.5 and $252 \mathrm{~nm}$, respectively). The higher size of the particles could be due to the aggregation of the particles within the dispersion medium.

To enhance the efficiency of the GTP, liposomal nanoparticles were used as carriers. Upon loading different concentrations of the GTP to the liposomal nanoparticles, with an increased concentration of the GTP up to $5 \mathrm{mg} \cdot \mathrm{mL}^{-1}$, there was an increase in the EE and on further increasing, the GTP concentration, EE was decreased (Table 1). Therefore, $5 \mathrm{mg} \cdot \mathrm{mL}^{-1} \mathrm{GTP}$ was considered as an optimal loading concentration where EE was $77.7 \%$, while Ramasamy et al. (2014) have reported the EE of MTX hydrochloride to the liposomal nanoparticles to be $\sim 60 \%$ (25). Analyzing the release pattern, by applying different loaded concentrations of the GTP showed no particular trend in the cumulative percentage of the drug release. The $\mathrm{pH}$ of the release medium was shown to play a role in the release of GTP from liposomal nanoparticles. A maximum of $60 \%$ of the GTP was released in $48 \mathrm{~h}$ at a $\mathrm{pH}$ of 7.4. In a similar study carried out by Ramasamy et al. (25), a maximum of $80 \%$ of the drug was released from the liposomal nanoparticles in $48 \mathrm{~h}$ when the release was carried out at $\mathrm{pH}$ 7.4. As the release medium shifted from acidic to basic, there was an increase in the cumulative percentage of the drug release. One of the limitations for the orally developed formulations is its metabolism in the gastrointestinal tract (GIT) which is mostly due to the harsh $\mathrm{pH}$ conditions of the GIT. In our study, to simulate the conditions of GIT, the release was carried out in a medium with acidic $\mathrm{pH}$ (pH 3). In an acidic $\mathrm{pH}(\mathrm{pH} 3)$, a lower percentage of the drug was released, while the maximum drug was retained within the nanoparticles. In basic $\mathrm{pH}$ (pH 9), the percentage of drug release was increased. The basic $\mathrm{pH}$ might have affected the structure or the complexity of the liposomes, thereby releasing more amount of the drug. The temperature of release medium was directly proportional to the cumulative percentage of the drug release. As the temperature was increased, there was an increase in the drug release percentage. The higher temperatures could influence the structure of the liposomal nanoparticles or may affect the binding between liposomes and GTP thereby releasing a higher percentage of the drug (Fig. 2).

In vitro, drug release data was fitted into various kinetics models to evaluate the drug release mechanism (Table 2). The ideal model of the drug release for nanoparticulate dosage forms or sustained release formulations is the Zero order kinetics (17). All our release data showed good correlation with Zero order kinetics, therefore, liposomal nanoparticles could be used as an ideal carrier for the delivery of GTP, achieving a prolonged or a sustained release. Korsmeyer-Peppas stated that for a Fickian release, $\mathrm{n}$ value is constrained to $0.5,0.45$, and 0.43 for the release from slabs, cylinders, and spheres (26). For spheres, values of $\mathrm{n}$ ranging from $0.43-0.89$ are regarded as anomalous and the release is characterized by diffusion and erosion mechanism and that $>0.89$ is characterized by the polymer erosion and relaxation and is termed as super case II transport. In our study, $\mathrm{n}$ values $\leq 0.43$ was regarded as a pure Fickian release, as the release of GTP was from spherical nanoparticles and the release was through diffusion. Predominance in anomalous transport was observed, which involves a coupling of erosion and diffusion.

Antioxidant assay revealed the potent scavenging 
activity of the GTP with respect to the released GTP at various times (Fig. 3). It has proved the retention of the antioxidant activity of GTP even after release from the liposomal nanoparticles. The highest scavenging activity was observed between 5-7 h which corresponds to the higher amounts of drug released. The potent scavenging activity was observed for all the released samples and GTP encapsulated liposomal nanoparticles could be used as an effective antioxidant source.

To conclude, for achieving a prolonged release of the GTP, 5 mg. $\mathrm{mL}^{-1}$ loading concentration was ideal in the acidic $\mathrm{pH}$ at $37^{\circ} \mathrm{C}$. The in vitro release data showed a good correlation of the GTP released with the release parameters such as $\mathrm{pH}$ and temperature, suitable for an ideal oral formulation where the release was minimum in the lower $\mathrm{pH}$ and temperature. The predominance was observed in Zero order kinetics with anomalous mode of transport. The antioxidant potential was observed for all the released samples. On further evaluation, the GTP encapsulated liposomal nanoparticles would serve as a potent source for oral delivery.

\section{Acknowledgement}

Authors would like to thank the management of VIT University for providing the required facility for carrying out the present work.

\section{References}

1. Fan Y, Zhang Q. Development of liposomal formulations: From concepts to clinical investigations. Asian J Pharm Sci. 2013; 8(2): 81-87. DOI: 10.1016/j.ajps.2013.07.010

2. Meunier F, Prentice HG, Ringden O. Liposomal amphotericin B (AmBisome): safety data from a phase II/III clinical trial. J Antimicrob Chemother. 1991; 28(1): 83-91. DOI: 10.1093/ jac/28.suppl_B.83

3. Leonard RCF, Williams S, Tulpule A, Levine AM, Oliveros S. Improving the therapeutic index of anthracycline chemotherapy: Focus on liposomal doxorubicin (Myocet ${ }^{\mathrm{TM}}$ ). Breast. 2009; 18(4): 218-224. DOI: 10.1016/j.breast.2009.05.004

4. Sarris AH, Hagemeister F, Romaguera J, Rodriguez MA, McLaughlin P, Tsimberidou AM, et al. Liposomal vincristine in relapsed non-Hodgkin's lymphomas: Early results of an ongoinig phase II trial. Ann Oncol. 2000; 11(1): 69-72. DOI: 10.1023/A:1008348010437

5. Phuphanich S, Maria B, Braekman R, Chamberlain M. A pharmacokinetic study of intra-CSF administered encapsulated cytarabine $\left(\right.$ DepoCyt $\left.t^{\circledR}\right)$ for the treatment of neoplastic meningitis in patients with leukemia, lymphoma, or solid tumors as part of a phase III study. J Neuro Oncol. 2007; 81(2): 201-208. DOI: 10.1007/s11060-006-9218-X

6. Ong HX, Benaouda F, Traini D, Cipolla D, Gonda I, Bebawy $\mathrm{M}$, et al. In vitro and ex vivo methods predict the enhanced lung residence time of liposomal ciprofloxacine formulations for nebulisation. Eur J Pharm Biopharm. 2014; 86(1): 83-89. DOI: 10.1016/j.ejpb.2013.06.024

7. Zhou X, Wang J, Wu J, Yang X, Yung BC, Lee LJ, et al.
Preparation and evaluation of a novel liposomal formulation of cisplantin. Eur J Pharm Sci. 2015; 66(1): 90-95. DOI: 10.1016/j. ejps.2014.10.004

8. Chen Z, Zhu QY, Tsang D, Huang Y. Degradation of green tea catechins in tea drinks. J Agric Food Chem. 2001; 49(1): 477482. DOI: $10.1021 / \mathrm{jf000877h}$

9. Lambert JD, Yang CS. Cancer chemopreventive activity and bioavailability of tea and tea polyphenols. Mutat Res. 2003; 523-524: 201-208. DOI: 10.1016/S0027-5107(02)00336-6

10. Hirano R, Momiyama Y, Takahashi R, Taniguchi H, Kondo $\mathrm{K}$, Nakamura $\mathrm{H}$, et al. Comparison of green tea intake in Japanese patients with and without angiographic coronary artery disease. Am J Cardiol. 2002; 90(10): 1150-1153. DOI: 10.1016/S0002-9149(02)02787-X

11. Mandel S, Amit T, Reznichenko L, Weinreb O, Youdim MBH. Green tea catechins as brain-permeable, natural iron chelatorsantioxidants for the treatment of neurodegenerative disorders. Mol Nut Food Res. 2006; 50(2): 229-234. DOI: 10.1002/ mnfr.200500156

12. Hong J, Lambert JD, Lee S, Sinko PJ, Yang CS. Involvement of multidrug resistance-associated proteins in regulating cellular levels of (-)-epigallocatechin-3-gallate and its methyl metabolites. Biochem Bioph Res Co. 2003; 310(1): 222-227. DOI: 10.1016/j.bbrc.2003.09.007

13. Lu H, Meng X, Li C, Sang S, Patten C, Sheng S, et al. Glucuronides of tea catechins: enzymology of biosynthesis and biological activities. Drug Metab Dispos. 2003; 31(4): 452-461. DOI: $10.1124 / \mathrm{dmd} .31 .4 .452$

14. Guan T, Miao Y, Xu L, Yang S, Wang J, He H, et al. Injectable nimodipine-loaded nanoliposomes: Preparation, lyophilisation and characteristics. Int $J$ Pharmaceut. 2011; 410(1-2): 80-87. DOI: 10.1016/j.ijpharm.2011.03.009

15. Swain T, Hillis WE. The phenolic constituents of Prunus domestica. I. - The quantitative analysis of phenolic constituents. J Sci Food Agric. 1959; 10(1): 63-68. DOI: 10.1002/jsfa.2740100110

16. Williams WR, Cuvelier ME, Berset C. Use of a free radical method to evaluate antioxidant activity. LWT-Food Sci Technol. 1995; 28(1): 25-30. DOI: 10.1016/S0023-6438(95)80008-5

17. Costa P, Lobo JMS. Modeling and comparison of dissolution profiles. Eur J Pharm Sci. 2001; 13(2): 123-133. DOI: 10.1016/ S0928-0987(01)00095-1

18. Allen TM, Cullis PR. Liposomal drug delivery systems: From concepts to clinical applications. Adv Drug Deliver Rev. 2013; 65(1): 36-48. DOI: 10.1016/j.addr.2012.09.037

19. Dhule SS, Penfornis P, Frazier T, Walker R, Feldman J, Tan G, et al. Curcumin-loaded $\gamma$-cyclodextrin liposomal nanoparticles as delivery vehicles for osteosarcoma. Nanomed-Nanotechnol. 2012; 8(4): 440-451. DOI: 10.1016/j.nano.2011.07.011

20. Helbok A, Rangger C, von Guggerberg E, Saba-Lepek M, Radolf $\mathrm{T}$, Thurner G, et al. Targeting properties of peptide-modified radiolabeled liposomal nanoparticles. Nanomed-Nanotechnol. 2012; 8(1). 112-118. DOI: 10.1016/j.nano.2011.04.012

21. Smith A, Giunta B, Bickford PC, Fountain M, Tan J, Shytle RD. Nanolipidic particles improve the bioavailability and $\alpha$-secretase inducing ability of epigallocatechin-3-gallate (EGCG) for the treatment of Alzheimer's disease. Int J Pharmaceut. 2010; 389(1-2): 207-212. DOI: 10.1016/j.ijpharm.2010.01.012

22. Santhosh PB, Kiryakova SI, Genova JL, Ulrih NP. Influence of iron oxide nanoparticles on bending elasticity and bilayer fluidity of phosphotidylcoline liposomal membranes. Colloids 


\section{Upputuri RTP \& Mandal AKA}

and Surfaces A: Physicochem Eng Aspects, 2014; 460: 248-253. DOI: 10.1016/j.colsurfa.2014.02.035

23. Wang Y, Zhang L, Guo S, Hatefi A, Huang L. Incorporation of histone derived recombinant protein for enhanced disassembly of core-membrane structured liposomal nanoparticles for efficient siRNA delivery. $J$ Control Release. 2013; 172: 179189. DOI: 10.1016/j.jconrel.2013.08.015

24. Doane TL, Chuang CH, Hill RJ, Burda C. Nanoparticle zetapotentials. Acc Chem Res. 2011; 45: 317-326. DOI: 0.1021/ ar200113c
25. Ramasamy T, Haidar ZS, Tran TH, Choi JY, Jeong J, Shin BS, et al. Layer-by-layer assembly of liposomal nanoparticles with PEGylated polyelectrolytes enhances systemic delivery of multiple anticancer drugs. Acta Biomater. 2014; 10(12): 51165127. DOI: 10.1016/j.actbio.2014.08.021

26. Martinez A, Iglesias I, Lozano R, Teijon JM, Blanco MD. Synthesis and characterization of thiolated alginate-albumin nanoparticles stabilized by disulfide bonds. Evaluation as drug delivery systems. Carbohyd Polym. 2011; 83(3): 1311-1321. DOI: 10.1016/j.carbpol.2010.09.038 